\title{
A Hardware Runtime for Task-based Programming Models
}

\author{
Xubin Tan, Jaume Bosch, Carlos Álvarez, Daniel Jiménez-González, Eduard Ayguadé, \\ and Mateo Valero, Fellow, IEEE
}

\begin{abstract}
Task-based programming models such as OpenMP 5.0 and OmpSs are simple to use and powerful enough to exploit task parallelism of applications over multicore, manycore and heterogeneous systems. However, their software-only runtimes introduce relevant overhead when targeting fine-grained tasks, resulting in performance losses. To overcome this drawback, we present a hardware runtime Picos++ that accelerates critical runtime functions such as task dependence analysis, nested task support, and heterogeneous task scheduling. As a proof-of-concept, the Picos++ hardware runtime has been integrated with a compiler infrastructure that supports parallel task-based programming models. A FPGA SoC running Linux OS has been used to implement the hardware accelerated part of Picos++, integrated with a heterogeneous system composed of 4 symmetric multiprocessor (SMP) cores and several hardware functional accelerators (HwAccs) for task execution. Results show significant improvements on energy and performance compared to state-of-the-art parallel software-only runtimes. With Picos++, applications can achieve up to 7.6x speedup and save up to $90 \%$ of energy, when using 4 threads and up to 4 HwAccs, and even reach a speedup of 16x over the software alternative when using $12 \mathrm{HwAccs}$ and small tasks.
\end{abstract}

Index Terms-Fine-grained parallelism; Task-dependence analysis; Nested tasks; Heterogeneous task scheduling; Energy saving; FPGA; Task-based programming models;

\section{INTRODUCTION}

$\mathrm{P}$ ARALLEL computing has become an ubiquitous principle to gain performance on multicore and manycore platforms. However, it exposes significant challenges, such as detecting parallel regions, distributing tasks/works evenly and synchronizing them. Furthermore, current processor architectures evolve towards more heterogeneity which only aggravates the aforementioned difficulties. Taskbased programming models are quickly evolving to target these challenges. Significant examples are OpenMP 5.0 [1], OmpSs [2], etc. Using these programming models, an application can be expressed as a collection of tasks with dependences, whose execution orders and potential data movements are enforced through dynamically constructing their task dependency graph (TDG) in runtime. Although with moderate size tasks, those programming models are able to exploit high levels of task parallelism with their default software-only runtimes, with fine-grained tasks, they suffer different degrees of performance degradation due to the runtime overhead and thread contention [3], [4], [5].

A straightforward way to overcome this deficiency and enable a finer task parallelism is to improve the overall runtime by accelerating its most critical functions in hardware. For homogeneous multicores, task dependence analysis is the most critical function [5], [6], [7]. For heterogeneous architectures, the task scheduling cost is also very high due to the load balance challenge caused by the execution time difference, the necessary data movements and synchronization between different memories [8], [9], [10]. Previous works of hardware task dependence management

- All authors are with the Barcelona Supercomputing Center (BSC) and Universitat Politècnica de Catalunya (UPC).

E-mail:name.surname@bsc.es, \{calvarez,djimenez\}@ac.upc.edu have showed great scalability and performance improvement over their software-only alternatives [4], [11], [12], [13]. However, firstly these works did not support nested tasks, that is, tasks that have been created by another task; secondly they did not support task scheduling to different hardware in heterogeneous systems; finally they have not been evaluated in a real system integrated as a hardware support within a programming model runtime. The most comprehensive analysis of a hardware task dependence manager [14] up to the moment was done targeting a small system with only two cores presenting good performance results but with a very limited scope.

In this paper we make a thorough analysis of Picos++, a general purpose hardware for task dependence management, nested task support and heterogeneous task scheduling for task-based programming models. The main contributions of this paper are as follows:

- A new heterogeneous task scheduling support. For the first time a hardware task scheduler is able to schedule tasks to different hardware execution units (SMPs and hardware accelerators).

- A novel hardware/software co-design for supporting nested tasks. The original version developed for SMP only systems has been extended to prevent deadlocks when the heterogeneous hardware resources are fully used.

- Picos++ implementation fully integrated with a taskbased programming model in a heterogeneous FPGA system running Linux OS and being able to execute problems concurrently in both FPGA and SMP.

- Detailed scalability and energy consumption studies during real executions of applications compar- 
Listing 1: multisort with OpenMP directives

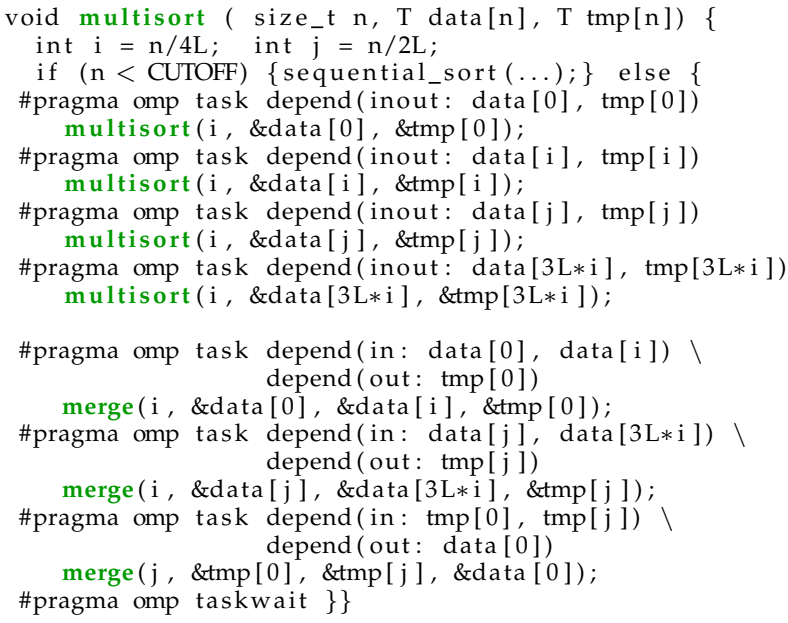

ing both Picos++ and software-only runtime systems, where tasks are executed in both threads and HwAccs. For the first time the real executions is extended to up to 15 execution units, providing insight of the impact of the hardware runtime with more hardware devices in the system.

- Execution trace support for application and Picos++ analysis.

The paper is organized as follows: Section 2 describes the background and previous work. Section 3 presents the Picos++ system. In Section 4, the experimental setup and benchmarks are described. Section 5 presents detailed studies of the impact of Picos++ on performance and energy consumption. Finally, in Sections 6 and 7 we discuss related work and conclude this paper.

\section{BACKGROUND}

\subsection{Task-based Programming Model}

OpenMP provides a powerful way of annotating sequential programs with directives to exploit task parallelism. For example in $\mathrm{C} / \mathrm{C}++$ language: \#pragma omp task depend(in: ...) depend(out: ...) depend (inout: ....) is used to specify a task with the direction of its data dependences (scalars or arrays). Implicit synchronization between tasks is ensured by the task dependence analysis in runtime. Explicit synchronization is managed by using \#pragma omp taskwait, which makes a thread wait until all its child tasks finish before it can resume the code execution.

An example of multisort source code with OpenMP annotations is shown in Listing 1 . Each multisort task instance can create four child multisort tasks and three child merge tasks as shown in lines 4-21. When the compiler finds a task annotation in the program it outlines the next statement and introduces a runtime system call to create a task (represented as a Task Descriptor). At execution time, the runtime system manages task creation, computes TDG, and schedules tasks when all their dependences are ready. The taskwait pragma at line 22 will wait for all the child tasks to finish.
Listing 2: Matmul block functions with OmpSs annotation

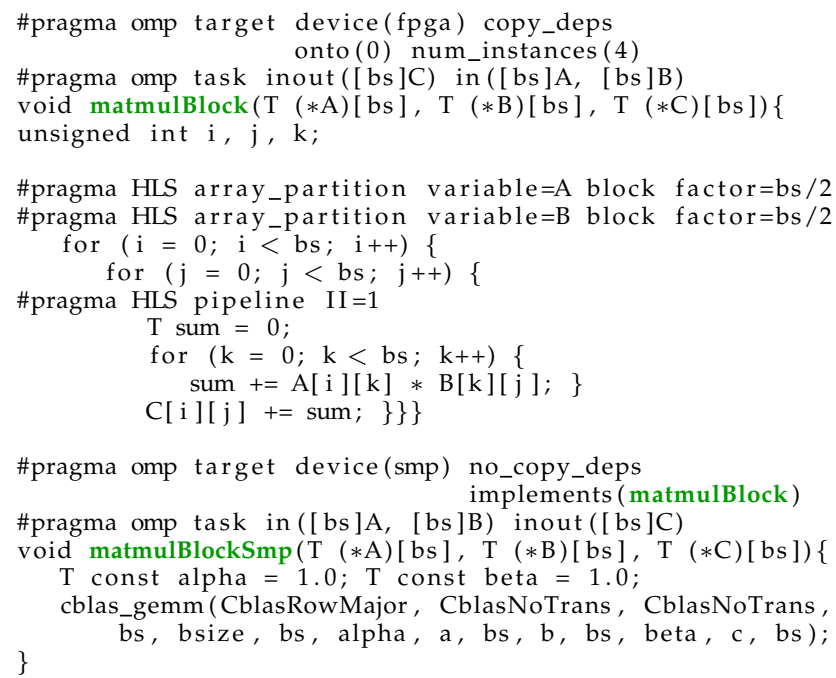

\subsubsection{Nested tasks}

The aforementioned child tasks are also named nested tasks in the OpenMP standard. They are supported to improve the programmability and nested parallelism. For instance, a nested task can be found in recursive code where the recursive function is a task that can be further decomposed (like in Multisort), or even when using tasks to call libraries with embedded tasks. Therefore, nested task support is a necessary feature of any task manager that wants to execute general-purpose code. Besides the fact that a parent task has to wait for all its child tasks, there are two more definitions that are important to consider: 1) A "child task" can only have a subset of its parent task's dependences. 2) The dependences of a "child task" only affect its sibling tasks ("child tasks of the same parent").

\subsubsection{Heterogeneous Task Scheduling}

The OmpSs programming model is developed as a forerunner for the OpenMP standard. In this work we will use it as an example for heterogeneous task scheduling. Listing 2 shows an example of OmpSs application with two instances of Matmul block functions that can be executed in either HwAccs in FPGA or in SMP threads. For example, the matmulBlock function in line 4 is annotated with both task and target device pragmas. The task pragma indicates the data dependences. The target device indicates that this task is going to be executed in the FPGA. At compile time, 4 instances of type $0 \mathrm{HwAcc}$ for this function are created as indicated by onto(0) num_instances(4) clause. In addition, the copy_deps specifies to copy the task dependence data from the host memory to the HwAccs (for input data) and from the HwAccs to the memory (for output data). The source-to-source Mercurium compiler generates the SMP code with the runtime (Nanos++) calls, task function code for SMP tasks, and the HwAccs for FPGA tasks [15]. Gcc compiler generates the binary code of the SMP, and the Xilinx tools Vivado and Vivado HLS, generate the bitstream of the FPGA. 


\subsection{Picos Overview}

The Picos hardware design [13] started with the aim to accelerate task dependence management for task-based programming models to exploit fine-grained task parallelism. From the software point of view, it can be seen as a blackbox that (1) reads new tasks and dependences from memory at task creation time; (2) writes ready-to-execute tasks in memory for the worker threads and (3) processes finished tasks.

The first hardware prototype of Picos [4] has a similar architecture to Picos++, shown in Figure 1. Picos is composed of five main components. Gateway (GW) fetches new and finished tasks from outside, and dispatches them to Task Reserve Station (TRS) and Dependence Chain Tracker (DCT). TRS is the major task management unit. It stores inflight tasks, tracks the readiness of new tasks and manages the deletion of finished tasks. It includes a Task Memory (TM) with 256 entries for up to 256 in-flight tasks, not shown in the figure. Each entry stores the task identification (TaskID), the number of dependences per task (\#Deps), the position in the DCT where each dependence is saved, and a reference to the next task consumer of each dependence. DCT is the major dependence management unit. It manages task dependences through one Dependence Memory (DM) and one Version Memory (VM). DM stores the memory addresses of dependences as tags and performs address match for each new dependence to these arrived earlier, to track data dependences. VM keeps the last consumer and the chain of producers of all the references to the same dependence address (called versions). Arbiter (ARB) manages the communication between TRS and DCT. Task Scheduler (TS) stores all ready tasks and schedules them to idle workers. Each pair of components are connected with FIFOs to ensure asynchronous communications.

Picos uses synchronous communication [4] between software and hardware. In fact, due to the communication mechanism the overall system performance is burdened. Threads are in charge of program- ming the DMA for each data movement (new, ready, finished task) between main memory and Picos. Although simple, this method is costly due to the frequent and small data exchanges in the system.

\subsubsection{Hardware task scheduling applicability}

Although Picos and Picos++ (as will be explained) have been implemented in a system composed of (few) ARM cores and an FPGA, this implementation aims to be only a proof-of-concept of its usability and capabilities. Picos and Picos++ are designed to work in a different scenario from the one presented. Such scenario is a multicore system of any type of SMP processors (i.e. ARM, Risc-V, Intel, IBM processors, etc.), where Picos++ would be implemented as an ASIC connected to all the cores. In such a system, Picos++ will run at the same frecuency as the cores and manage even more fine grained tasks (as the communication will be faster that in the current implementation). Such system is expected to obtain even higher benefits from hardware task scheduling than the ones obtained in the current paper. If the system is in addition composed of some heterogeneous components (such different SMP cores, FPGAs or GPUs or any other type) Picos++ ASIC will also take care of

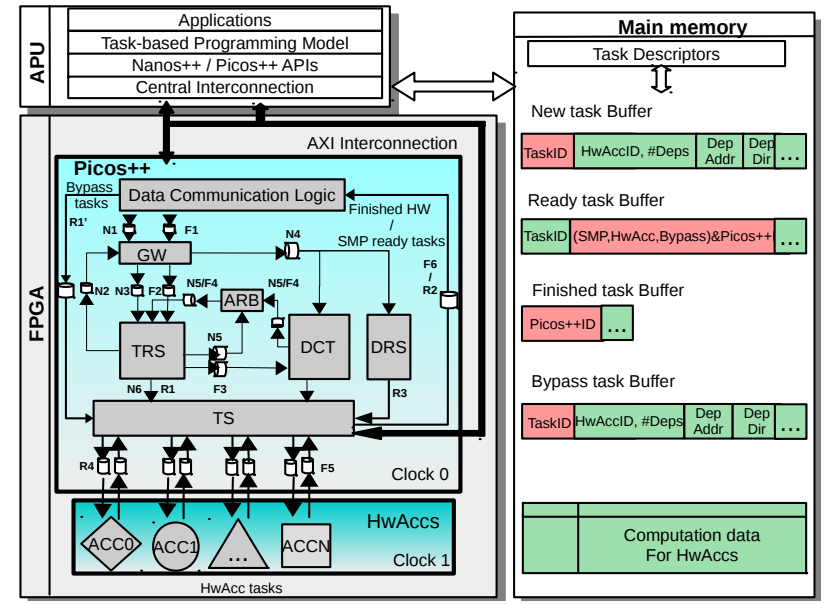

Fig. 1: Picos++ system organization

this components. In current environments, the SMP threads are the ones in charge of managing the accelerators and this work also adds overheads that Picos++ ASIC would mitigate.

The same could be said about the programming model used. Although in this paper we target OmpSs as a programming model that already has good support for FPGA task execution, any task based programming model would benefit from Picos++. The API of Picos++ for receiving a task with dependences, releasing ready tasks and receiving notitifications of finished tasks is general enough to be adapted to other task-based programming models runtimes (e.g. OpenMP runtimes) and even to other programming models. In this sense, the aim of this work is to prove that the task scheduler is one the next components that should be moved from software to hardware in future processors.

\section{Picos++ System}

Picos++ is an evolution of the previous design with several important new features introduced to support nested tasks, tasks with unlimited number of dependences and heterogeneous task scheduling. The main new components in Picos++ not included in the previous Picos design showed in figure 1 are: the Bypass task buffer stored in main memory for communication, the Dependence Reserve station (DRS) module in Picos ++ , the TS communication with the hardware accelerators (HwAccs) and the HwAccs themselves. In addition to those, the modifications also include a new hardware-software asynchronous communication scheme [14] and different GW, DCT and TS behaviors, including a new DCT memory as described in section 3.2.

Figure 1 shows the sequence of new (labeled N\#) and finished task (labeled F\#) processing. The ready task processing (labeled R\#) will be introduced later. When a new task arrives, GW reads the task and its dependences (N1) and requests a free TM entry from TRS (N2). Afterwards, it distributes the task to TRS (N3) and its dependences to DCT (N4). TRS saves the task information including TaskID and \#Deps in the corresponding TM entry. DCT performs address match of each dependence to those arrived earlier. If there is no match, DCT notifies TRS that this dependence is ready (N5). Otherwise, there is a possible producer or consumer match, DCT saves the producer match and only 


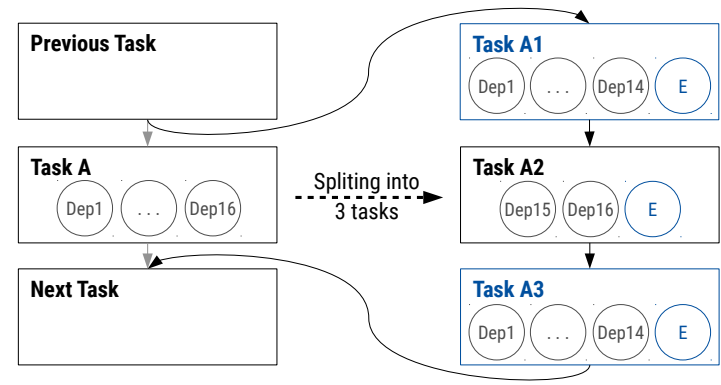

Fig. 2: Process for managing tasks with more than 15 dependences

notifies TRS of the consumer match (N5). By doing this, for all the producer-consumer chain (aka. true-date dependence chains), the producer is saved in DCT while the consumers are saved in TRS. TRS processes all these notifications. For each ready one, TRS checks if all the dependences of this task are resolved; if so, TRS marks it ready and schedules it either to an idle thread or HwAcc (N6) meanwhile TRS forwards a ready message to the next consumer task (if it exists) dependent on this ready dependence (also N5). Otherwise TRS saves this possible consumer match. When a finished task arrives, GW reads (F1) and forwards it to TRS (F2). TRS checks the corresponding TM entry for all the dependences and sends finished notification of them to DCT (F3). DCT checks DM and VM for each finished dependence to either delete its instances or send ready notifications to TRS to wake up the dependences that were previously not ready $(\mathrm{F} 4)$.

Figure 1 also shows the Picos++ system implementation. It mainly consists of the application processing unit (APU) with SMP inside, a FPGA and a shared main memory. Picos++ and HwAccs are inside the FPGA, using clock domains 0 and 1 respectively. All the data exchange between these two clock domains are dealt with either asychronous FIFOs or clock-crossing (standard method to pass multibits or 1-bit data safely from one clock domain to another).

There are two sets of communications: first is for new, ready, finished and bypass tasks, where Picos++ and APU share them in the main memory; second is the computation data required by the HwAccs. Each HwAcc used is capable of reading input data from the main memory, does computation and then writes back results to the main memory, without help from the SMP threads inside APU.

\subsection{Hardware/Software Synchronization}

For the task communication between SMP threads and Picos++ in FPGA, there are four circular buffers, one for each type of tasks shown in Figure 1. Each buffer can store up to $\mathrm{N}$ units representing $\mathrm{N}$ tasks. At the beginning of the application execution, the software side of the runtime starts Picos++ and allocates those buffers in main memory. Afterwards, it sends the buffer addresses and lengths to Picos++. When the application finishes, it deallocates the buffers and stops Picos++ [14].

New task buffer stores new task information required by Picos++. Each new task entry consists of: TaskID (8 bytes), HwAccID (2 bytes), \#Dependences (2 bytes), and for each dependence, the dependence memory address ( 8 bytes) and direction (4 bytes). HwAccID identifies which are the hardware devices where a particular task can be executed (currently, up to 16 different types).

The current hardware implementation supports a maximum of 15 dependences per task. In the case of more dependences, as this is known at compile time, the task can be splitted into an artificial chain of tasks with at most 15 dependences each. Figure 2 shows how an original task A with 16 dependences is split into three tasks A1, A2 and A3 that have 15 or less dependences per task and maintains the correct execution order. Task A1 will be created with 14 of the original dependences and an Extra (E) helper dependence with inout type. A1 will be a empty task that has no computation. Task A2 will have the remaining 15th and 16th original dependences and the same Extra (E) helper dependence with inout type. It will inherit all the computation from the original Task A. This way through the inout dependence chain, the original code will be executed only after all the original dependences are ready. Finally, another empty helper task A3 will do the same function as task A1. This last task will ensure that any task that depends on any of the 16 original dependences is executed in the correct order. This simple mechanism can be replicated for any number of dependences using more helper tasks. In fact, it allows Picos++ to process tasks with more dependences than fit in its internal memories (as the helper tasks dont need to be processed as a single unit) and maintains the parallel processing in a nested task environment.

Ready task buffer holds not only the ready-to-execute SMP tasks, but also messages that signal the software part of the runtime that a HwAcc has finished executing a ready task. Each unit includes TaskID (8 bytes), (SMP, HwAccs, Bypass) (3 bits) and Picos++ID (14 bits). For each SMP task (identified by the SMP bit), the software runtime schedules it to execute in threads; for each finished executing HW task (identified by the HwAcc or Bypass bit), the software runtime deletes the corresponding task descriptor in memory.

Finished task buffer stores the information (Picos++ID) of finished tasks from threads, to send it back into Picos++ for TDG update. Note that this is only for tasks scheduled to execute in threads. For tasks scheduled to execute in the HwAccs, they are directly sent to HwAccs and retrieved back through separate FIFO queues inside the FPGA. Therefore, those finished tasks would not appear in this buffer.

Bypass task buffer stores bypass tasks, with the same format as the new task buffer. The bypass tasks are already ready to execute. This concept is introduced to tackle two new situations raised by HwAccs. The first one is to handle applications that want to accelerate part of the code using the HwAccs without Picos++ task dependence manager. They need a direct communication mechanism from the APU to the FPGA that bypasses the dependence manager and directly executes in HwAccs. The second situation appears in applications with nested tasks. When all the Picos++ internal task dependence management resources are used up, and this channel allows us to continue executing tasks in HwAccs.

In the case of new, finished and bypass task buffers, SMP threads write to these buffers while Picos++ hardware reads them while in the case of the ready task buffer, the communication works in the opposite direction. For all the buffers, entry fields have valid bits to inform the Picos++ 


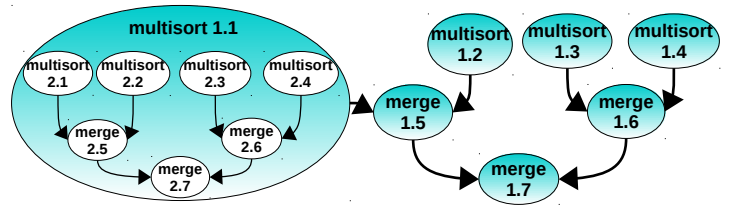

Fig. 3: Multisort with nested task dependences

hardware and software part that those entries are valid or not, and also the type of tasks.

The data communication between software and Picos++ hardware is important for the final system performance. Picos++ implements a buffered and asynchronous communication which requires small support from threads. It decouples the data communication between software and hardware, ensuring an efficient small data exchange suitable for our user case. As a result, Picos++ obtains a high overall system performance [14].

\subsection{Nested Task Support}

To support nested tasks, we add a new field parentID for each new task in the task buffer. This parentID together with taskID allow the dependences of non-sibling tasks to be maintained in separate TDGs inside Picos++.

Nonetheless, this is not enough to avoid corner cases that can lead to a whole system deadlock. Those corner cases derive from the fact that hardware task managers, opposed to the software ones, have a limited amount of memory. Thus, it is possible for them to read a task and get their internal memory full in the middle of processing task dependences. Without nested tasks, this is not a problem because previous processed tasks go to execution and eventually they finish and free out resources that will allow the hardware to proceed with the stalled tasks.

For nested tasks with dependences, the situation is much more complicated. To simplify and explain a possible deadlock scenario, assume that Picos++ has memory capacity for only 7 tasks. Figure 3 shows the TDG of the Multisort application when two levels of nested tasks have been reached. Tasks in level $\mathrm{i}$ are labeled i.X, where $\mathrm{X}$ is the number of the sibling task order creation. While all tasks in level 1 are shown, only child tasks of multisort 1.1 are shown for the second level. In this case, the first level tasks would quickly fill the Picos++ hardware manager when the executing multisort 1.1 tries to create new tasks. In particular, multisort 1.1 will create the first child task multisort 2.1 , that will get stuck due to a full memory problem. As a parent task multisort 1.1 has to wait until all its child tasks end (and they will not end), it cannot finish and all the following multisort (which can create similar child tasks) and merge $1 . x$ tasks, already inside the hardware manager, can not proceed (and free memory for more tasks), so the system goes into a deadlock.

\subsubsection{Deadlock Free Hardware/Software Co-design}

In order to support nested tasks without deadlocks, we introduce a hardware/software co-design in Picos++.

Atomic task processing is the hardware solution which offers the first step to avoid deadlocks by reading and processing tasks atomically. Once a task is read all its dependences should be processed (i.e. integrated in the
TDG). Using a non-atomic task processing would allow the GW to read new tasks without considering the free spaces in all the internal memories (TM, DM and VM) until the FIFOs connecting them are full. Without nested tasks, this method ensures efficient inter-component communication. However, it leads to deadlocks easily with nested tasks. For instance, multisort 2.1 shown in Figure 3 could get stuck in those FIFOs and cause a deadlock. Thus, in Picos++ the hardware is modified to read and process tasks as a whole, which means GW is conscious of the free spaces in all Picos++ memories before it reads a new task.

This awareness has two cases to consider: when the new task has no new dependences (all of them are versions of dependences already in the hardware) and when there are new dependences. For the first case, it is easy to ensure the processing of a new task and new versions of already known dependences as they can be stored in any place of their respective memories (TM and VM). In this case, one empty space in the TM and 15 empty spaces in the VM are enough to read a new task. For the second case, ensuring the processing of new dependences is more difficult as they are stored using a hash process in order to have a fast location mechanism in DM [4]. DM uses hashing and a 8way associative memory, a full-associative memory has been discarded due to its complexity and resource requirement. Therefore, a dependence may not be stored because the memory set that should store the dependence is full, independently of the whole DM usage. This problem can not be anticipated and results in a negligible slow-down without nested tasks, but with nested tasks may lead to a deadlock. To overcome it, Picos++ has a new fall-back memory that is able to store up to 15 dependences. This memory is used whenever a new dependence cannot be stored in DM. Once it is used, it raises the memory full signal. This signal stops the GW from reading more new tasks while allows it to continue processing the remaining dependences of the current task. As soon as DM has free space, the dependences are moved from the fall-back memory back to DM, so Picos++ resumes new task processing.

Buffered task recovery is the software solution to work together with atomic task processing to avoid deadlocks. As described in the previous paragraph, the GW will not read multisort 2.1 when there is no space inside Picos++, so multisort 2.1 is stored inside the new task buffer in the main memory. However, in order for the application to proceed, it has to be processed somehow. The key to solve the deadlock is that multisort 2.1 is the first nonexecuted child of its parent in the buffer. Due to the fact that child dependences are always a subset of their parent, the first non-executed child (previous sibling tasks have been executed and their dependences released) is always ready. This does not hold true for the subsequent ones. Therefore, if the first non-executed child is still in the new task buffer, it has to be processed (somehow) in order to avoid deadlocks. Otherwise, if it has reached the hardware there is not going to be a deadlock. In both cases, the remaining children of a task must remain in the queue waiting to be processed until the hardware processes them in order to avoid race conditions.

To fulfill this requirement, the software support of $\mathrm{Pi}$ cos++ keeps track of the entries in the new task buffer where 


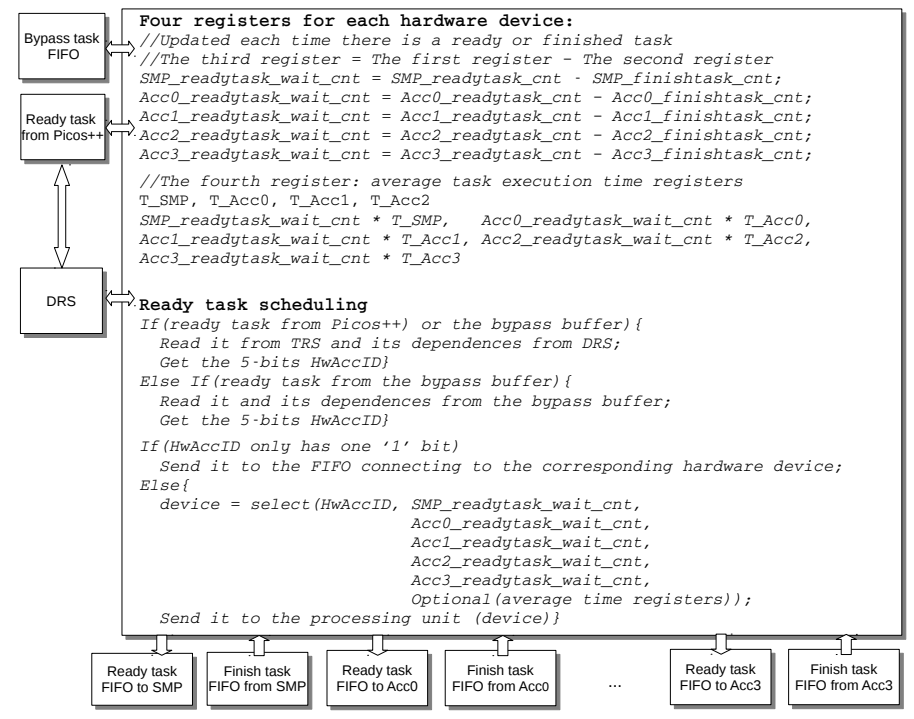

Fig. 4: Task scheduling to different hardware units

a parent has stored its children. Whenever a full condition arises, it checks the state of the first non-executed child. If it has been read, the software support stays at normal state. Otherwise the software support intervenes to avoid a possible deadlock. First, the thread locks the new task buffer and removes all its child tasks. Then, the buffer is reconstructed if it has any remaining tasks (created by other running tasks). Afterwards, either the child tasks removed are directly executed in order by the thread (without allowing them to create more tasks) or submitted as a whole to a software task dependence manager. Finally, when the full condition in hardware is cleared, the software support reverts to use hardware for dependence processing. The first option is simpler and keeps the complexity of the software part at bay. The second may allow extra parallelism to be extracted in some corner cases. In any case, tasks can be sent to HwAccs through the bypass task buffer.

\subsection{Heterogeneous task scheduling support}

To support task scheduling to up to 16 different hardware execution units, a new module called Dependence Reserve station (DRS) is introduced, in addition, the task scheduler (TS) module is modified.

\subsubsection{Dependence Reserve Station (DRS)}

As in Figure 1, DRS receives new dependence packets from GW. For each of them, DRS saves it into an internal memory indexed by the TRS entry address. When TS wants to schedule a task to a HwAcc, all the dependence memory addresses required can be read sequentially from DRS. This module allows easier and faster access for dependence addresses when scheduling tasks to HwAccs.

\subsubsection{Task Scheduler (TS)}

TS is mainly responsible for scheduling all the ready tasks to suitable hardware execution units to achieve an earliest finish execution time. Figure 4 shows a simplified scheme of the heterogeneous task scheduling algorithm in TS, assuming that there are one SMP and $4 \mathrm{HwAccs}$, that is, a total of 5 hardware units. Picos++ has one ready and one finished task queue for SMP, and one ready and finished task queue for each HwAcc. There are two sources of ready tasks: normal ones that are deemed ready by the Picos++ task dependence manager, and bypass tasks. TS checks alternatively from these two sources.

The TS has also four registers associated with each hardware device. Two registers count the number of ready tasks assigned to each HwAcc and the number of finished tasks from this unit. The third register gets the deduction of the previous two registers and indicates the total amount of work still waiting for this unit. Finally the fourth register counts the average task execution time.

Each ready task has a 5bits HwAccID generated by the target pragmas annotated on each function as shown in Listing 2. This HwAccID correponds to a mask that indicates in which accelerators the task can be executed (SMP and $\mathrm{HwAcc0}$ to $\mathrm{HwAcc} 3$ in figure 4). As shown in Figure 4, when a ready task arrives, TS first checks its hardware mask. If it's HwAccID indicates only a specific device, it will be scheduled directly to that device. Otherwise, TS compares and selects the one with the least number of waiting work. In the case of multiple devices with identical amount of waiting work, Picos++ selects the one with the higher priority either because this device has more memory bandwidth or is simply faster. The average execution time registers can be enabled when different hardware devices in the system have a big speed gap. In this prototype, the priorities of different hardware are fixed and are based on the infrastructure generated: the HwAcc3 has the highest and the SMP has the lowest priority. For systems that have different connections and hardware devices, the priorities can be modified to suit the characteristics of the system, thus achieving a better performance. This scheme has a small hardware cost, and it balances the workloads well among different hardware devices.

In Figure 1, for each ready task from TRS (R1), TS reads it and might schedule it to SMP (R2). If not, then TS reads all its dependences from DRS (R3) and schedules it to a HwAcc (R4). For a bypass task (R1'), TS gets all its dependences from the bypass task buffer in memory and schedules it to a HwAcc (R4). When a task in the HwAcc finishes, TS retrives it through FIFO queue (F5) to update the Picos++ internal TDG, and also notifies it to the SMP through the ready task buffer in the main memory (F6)

\section{Experimental Setup and Benchmarks}

\subsection{Experimental Setup}

Picos++ has been coded in VHDL and tested in a Xilinx hardware platform. Its communication logic and all the HwAccs have been coded in C with Vivado HLS directives. The final system designs have been synthesized with Vivado 2016.3. The hardware platform contains a Zynq Ultrascale+ MPSoC Chip XCZU9EG [16]. It includes the Application Processing Unit (APU) with 4 ARM Cortex-A53 cores at $1.1 \mathrm{GHz}$ and a FPGA operating at a frequency from 50 to $300 \mathrm{MHz}$, with 4GB DDR4 main memory.

The evaluation of Picos++ is done using the task-based parallel programming model OmpSs [2], it is supported by the source-to-source Mercurium compiler and the Nanos++ runtime system. In this paper, the Picos++ runtime uses the 
same task creation mechanism as the Nanos++ runtime, but uses hardware for task dependence analysis and scheduling. We also use performance tools Extrae and Paraver [17] to analyze the application behavior in our system.

Sequential and parallel execution time of OmpSs applications are obtained in the system running Ubuntu Linux 16.04. Each execution has been run for 5 times, and the median value is used.

\subsection{Benchmarks}

\subsubsection{Synthetic and Real Benchmarks}

A brief description of the benchmarks follows:

TestFree creates $\mathrm{N}$ tasks with $\mathrm{M}$ dependences. Each task has the same execution time $\mathrm{T}$ and all of them can be executed in parallel (tasks are independent of each other). This benchmark is designed to illustrate the processing capacity of Picos++ in the case of an application with embarrassing parallelism.

TestChain creates $\mathrm{N}$ tasks with $\mathrm{M}$ dependences, each of execution time T. Each task is the consumer of the previous task, and the producer of the next task. This benchmark is designed to show the worst case, as it has no parallelism at all and suffers the communication latency for offloading task dependences to hardware.

TestNested creates 16 parent tasks. The first 15 tasks have 2 dependences per task but they are independent from each other. Each task (parent task) creates an inout chain of $M$ child tasks, and each child task can be configured to create its own nested tasks. This inout chain is implemented by using the first dependence of each parent. The 16th parent task has 15 dependences that depend on all the previous 15 parent tasks by using their second dependence. This benchmark with configurable nesting levels of tasks and configurable child tasks per parent is constructed to test the nested task support.

Cholesky Factorization computes $\mathrm{A}=\mathrm{LL}^{\prime}$, with $\mathrm{A}$ an $n \times n$ matrix and L lower-triangular.

Multisort sorts the input arrays using the divide and conquer method as can be seen in Listing 1 .

Matmul is matrix multiplication. It calculates the multiplication of two matrices $C=A B$.

TABLE 1: The characteristics of real benchmarks

\begin{tabular}{|l|c|r|r|r|}
\hline Name & Configs & \#Tasks & Seq time(us) & Latency(us) \\
\hline \multirow{3}{*}{ Matmul } & $(2 \mathrm{~K}, 32)$ & 262144 & 6820850 & 26 \\
& $(2 \mathrm{~K}, 64)$ & 32768 & 5463956 & 167 \\
& $(2 \mathrm{~K}, 128)$ & 4096 & 5435528 & 1327 \\
\hline \multirow{2}{*}{ Cholesky } & $(2 \mathrm{~K}, 32)$ & 45760 & 1232664 & 27 \\
& $(2 \mathrm{~K}, 64)$ & 5984 & 1087485 & 182 \\
\hline \multirow{2}{*}{ Multisort } & $(1 \mathrm{M}, 256,256 \mathrm{k})$ & 9565 & 395594 & 41 \\
& $(2 \mathrm{M}, 512,512 \mathrm{~K})$ & 9565 & 832882 & 87 \\
& $(1 \mathrm{M}, 1 \mathrm{~K}, 512 \mathrm{~K})$ & 2397 & 407648 & 170 \\
\hline
\end{tabular}

For real benchmarks [18], Table 1 shows the number of tasks, sequential execution time and task latency (granularity) in microseconds of the applications evaluated with different problem and block size. In the case of Multisort application, column Configs indicates the problem, minimal sort and merge size. During sequential and parallel execution, the non-recursive tasks of Matmul and Cholesky that are executed in threads use Openblas. In the case of Multisort, the non-recursive basic sort tasks are executed in threads using libc qsort.
TABLE 2: Characteristics of HwAccs in XCZU9EG

\begin{tabular}{|l|llll|l|}
\hline \multirow{2}{*}{ Name } & \multicolumn{4}{|c|}{ HWACCs } & Latency \\
\cline { 2 - 5 } & B_18Kb & DSP48E & FFs & LUTs & us \\
\hline fgemm32 & $68 / 3.7 \%$ & $160 / 6.4 \%$ & $19771 / 3.6 \%$ & $15559 / 5.7 \%$ & 27 \\
fsyrk32 & $36 / 2.0 \%$ & $160 / 6.4 \%$ & $19822 / 3.6 \%$ & $16149 / 5.9 \%$ & 63 \\
ftrsm32 & $36 / 2.0 \%$ & $104 / 4.1 \%$ & $11482 / 2.1 \%$ & $10875 / 4.0 \%$ & 67 \\
fpotrf32 & $10 / 0.6 \%$ & $22 / 0.9 \%$ & $3487 / 0.6 \%$ & $3302 / 1.2 \%$ & 168 \\
fgemm64 & $74 / 4.1 \%$ & $160 / 6.4 \%$ & $23887 / 4.4 \%$ & $30032 / 11.0 \%$ & 126 \\
fsyrk64 & $42 / 2.3 \%$ & $160 / 6.4 \%$ & $23849 / 4.4 \%$ & $30727 / 11.2 \%$ & 270 \\
ftrsm64 & $42 / 2.3 \%$ & $250 / 9.9 \%$ & $28734 / 5.2 \%$ & $25753 / 9.4 \%$ & 314 \\
fpotrf64 & $28 / 1.5 \%$ & $22 / 0.9 \%$ & $3514 / 0.6 \%$ & $3350 / 1.2 \%$ & 981 \\
\hline fmatmul32 & $68 / 3.7 \%$ & $162 / 6.4 \%$ & $20106 / 3.7 \%$ & $14671 / 5.4 \%$ & 27 \\
fmatmul64 & $138 / 7.6 \%$ & $322 / 12.8 \%$ & $38770 / 7.1 \%$ & $27668 / 10.9 \%$ & 105 \\
fmatmul128 & $287 / 15.7 \%$ & $642 / 25.5 \%$ & $76147 / 13.9 \%$ & $54462 / 19.9 \%$ & 497 \\
\hline sort256 & $68 / 3.7 \%$ & 0 & $20106 / 3.7 \%$ & $14671 / 5.5 \%$ & 5 \\
sort512 & $138 / 7.6 \%$ & 0 & $38770 / 7.1 \%$ & $27668 / 10.1 \%$ & 26 \\
sort1024 & $159 / 8.7 \%$ & 0 & $47034 / 8.6 \%$ & $71124 / 26.0 \%$ & 92 \\
\hline
\end{tabular}

TABLE 3: Hardware Resource and Power Consumption

\begin{tabular}{|c|c|c|c|c|c|}
\hline \multirow[t]{2}{*}{ Name } & \multicolumn{4}{|c|}{ FPGA resource } & Power \\
\hline & BRAM18K1 & $\widehat{\mathrm{SP} 48 \mathrm{E}}$ & FFs & $\overline{\text { LUTs }}$ & Watts \\
\hline XCZU9EG & 1824 & 2520 & 548160 & 274080 & \\
\hline Picos++ & $87 / 5.0 \%$ & 0 & $5478 / 1.0 \%$ & $9793 / 4.0 \%$ & 0.07 \\
\hline Comm. Logic & $2 / 0 \%$ & 0 & $3421 / 0.6 \%$ & $4244 / 1.0 \%$ & 0.03 \\
\hline APU & & & & & Watts \\
\hline 4 ARM Cortex-A53s & - & - & - & - & 1.4 \\
\hline
\end{tabular}

\subsubsection{HW Functional Accelerators (HwAccs)}

Table 2 shows the hardware cost (absolute number of logics and percentage of FPGA used) of different HwAccs, and their latencies in microseconds for executing a task. All the accelerators are synthesized using $200 \mathrm{MHz}$ clock. In the Cholesky application there are four different HwAccs: fgemm, fsyrk and ftrsm and fpotrf that correspond to its four different kernels. Matmul and Multisort applications have only one type of HwAccs each, fmatmul and sort, respectively.

\subsection{Hardware Resource and Power Consumption}

Table 3 shows the on-chip resource utilization and power consumption of Picos++ on the Zynq Ultrascale+ XCZU9EG chip [16]. The resource usage is obtained from Vivado postimplementation reports. Picos++ and its communication logic use around 5\% of the FPGA resources. This is important as not only it is small in order to be feasible to integrate it into multicore processors with very low cost, but also it allows us to build a highly heterogeneous platform combining Picos++ with other HwAccs. The 4 ARM CortexA53 cores take around $93.3 \%$ of the whole chip power $(1.4 \mathrm{~W}$ out of $1.5 \mathrm{~W})$. Picos++ and its data communication logic only consume $0.1 \mathrm{~W}$.

The power consumption is measured through dedicated hardware registers in three main hardware parts: FPGA, APU and the main memory DDR4. Energy consumption is calculated by integrating the total power consumed by the three parts along the application execution time.

\section{Performance and Energy Consumption}

\subsection{Task and Dependence Repetition Rate}

Table 4 shows the task and dependence repetition rates (rrTask and rrDep) of the software-only runtime and Picos++ measured in $100 \mathrm{MHz}$ clock cycles. TestFree and TestChain with 65536 empty tasks (zero execution time) are used to avoid the influence of task execution time, 


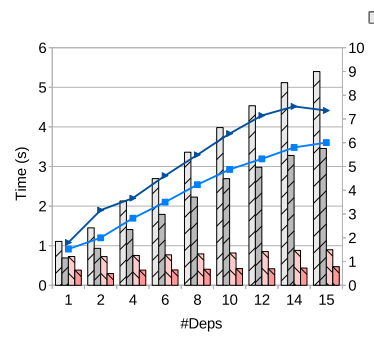

(a) TestFree

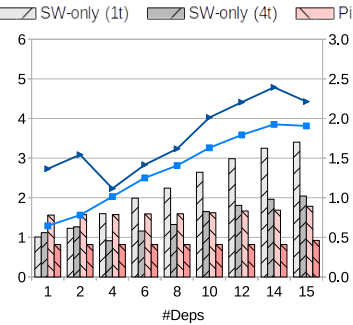

(b) TestChain

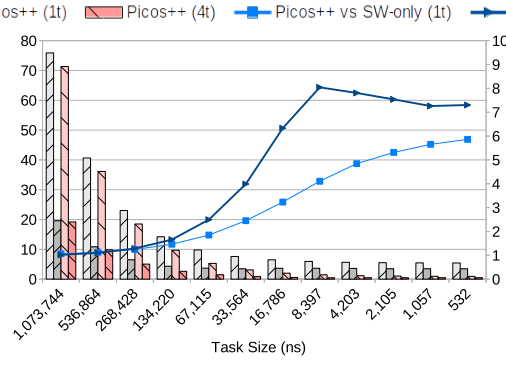

(c) TestFree 15 deps

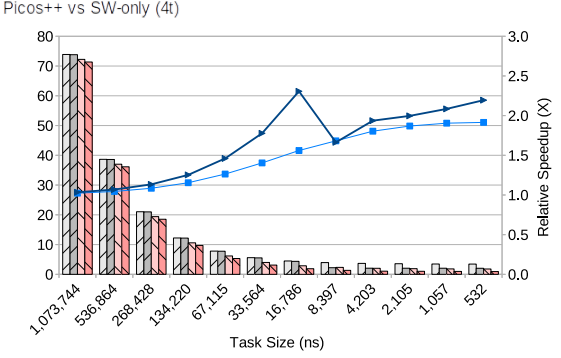

(d) TestChain 15 deps

Fig. 5: Execution time and relative speedup of TestFree, TestChain with 4 threads

TABLE 4: Task, Dependence Repetition Rate in cycles

\begin{tabular}{|c|c|c|c|c|c|}
\hline \multirow{2}{*}{\multicolumn{2}{|c|}{\begin{tabular}{|l|} 
Testcase \\
Number of dependences
\end{tabular}}} & \multicolumn{2}{|c|}{ TestFree } & \multicolumn{2}{|c|}{ TestChain } \\
\hline & & 1 & 15 & 1 & 15 \\
\hline \multirow{2}{*}{ HW-only } & rrTask & 24 & 243 & 35 & 348 \\
\hline & $\mathrm{rrDe}$ & 24 & 16 & 35 & 23 \\
\hline \multirow{2}{*}{ SW-only $2 \mathrm{t}$} & rrTask & \multirow{2}{*}{\multicolumn{2}{|c|}{$\begin{array}{l}11755281 \\
1175352\end{array}$}} & \multirow{2}{*}{\multicolumn{2}{|c|}{$\begin{array}{l}16683095 \\
1668206\end{array}$}} \\
\hline & rrDep & & & & \\
\hline \multirow{2}{*}{ SW-only 4t } & rrTask & \multirow{2}{*}{\multicolumn{2}{|c|}{$\begin{array}{l}10555272 \\
1055352\end{array}$}} & \multirow{2}{*}{\multicolumn{2}{|c|}{$\begin{array}{l}17063118 \\
1706208\end{array}$}} \\
\hline & rrDep & & & & \\
\hline Picos $++2 t$ & $\begin{array}{l}\text { rrTask } \\
\text { rrDen }\end{array}$ & $\begin{array}{l}731 \\
731\end{array}$ & $\begin{array}{l}855 \\
57\end{array}$ & \multicolumn{2}{|c|}{$\begin{array}{l}12511419 \\
125196\end{array}$} \\
\hline \multirow{2}{*}{ Picos $++4 \mathrm{t}$} & rrTask & 582 & 716 & \multirow{2}{*}{\multicolumn{2}{|c|}{$\begin{array}{l}12501406 \\
125094\end{array}$}} \\
\hline & rrDep & 582 & 48 & & \\
\hline
\end{tabular}

and different number of dependences are used to study its impact. Row HW-only shows results without any communication latencies or software runtime overhead (task creation and scheduling) in Picos++. Note that the communication between the processors and Picos++ costs around 300 clock cycles for the full life cycle of a task. Row SW-only $2 t$ and $4 \mathrm{t}$ show results obtained from real executions by using SWonly runtime with 2 and 4 threads. Similarly Row Picos++ $2 t$ and $4 t$ shows real execution results obtained by using the Picos++.

With HW-only, each task and dependence only takes a few cycles to be processedand only increases slightly when increasing the number of dependences. For example, tasks with 1 dependence in TestFree and TestChain are processed in 24 and 35 cycles respectively while with 15 dependences, they are processed in 243 and 348 cycles. The dependence repetition rate is quite steady for different dependence patterns ranging from 16 to 23 cycles. This is due to the fact that Picos++ pipelines the processing of all the dependences of a task. Tasks that are processed through the bypass task buffer have the same repetition rate as TestFree tasks with no dependences.

Althouth data communication has a big impact over system performance as it can be seen in Picos++ rows in Table 4, the two observations about HW-only hold true for Picos++ with $2 t$ and $4 t$. As a result, there is a clear speed up of managing task dependence analysis in hardware than in software.

\subsection{Synthetic Benchmarks}

\subsubsection{Performance Impact of the Number of Dependences}

Figure 5 shows results of TestFree and TestChain. All the figures have two Y-axis, the left one (in bars) indicating the execution time in seconds, and the right one (in lines) showing the relative speedup of Picos++ against the software-only runtime with 1 or 4 threads. The left two diagrams in Figure 5 display these two benchmarks with 65536 empty tasks

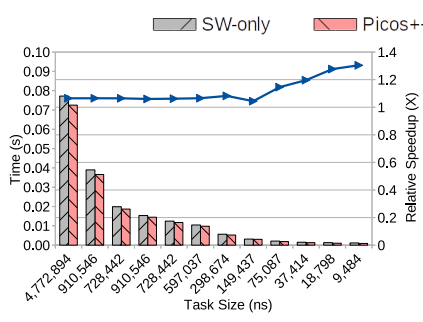

(a) 4 child in each parent task

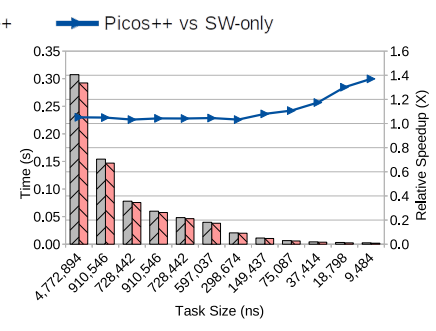

(b) 16 child in each parent task
Fig. 6: Time and speedup of TestNested with 4 threads

(tasks with 0 execution time), and with different number of dependences. Picos++ and SW-only are using the same task creation and scheduling mechanism, therefore with empty tasks the results highlight the dependence analysis cost difference and the communication overhead in Picos++. There are two key observations. First, Picos++ maintains a nearly equal performance from 1 to 15 dependences per task. Second, it has a much lower dependence analysis cost. For 15 dependences and TestFree, where there is full parallelism, Picos++ is $7.5 \mathrm{x}$ faster than SW-only using 4 threads. In the case of the TestChain, for the same number of dependences and where there is no parallelism at all, Picos++ still is up to $2 x$ faster than the SW-only runtime.

\subsubsection{Performance Impact of the Task Granularity}

The two right diagrams of Figure 5 show results for the same number of tasks, 15 dependences per task and a range of task sizes (X-axis) going down from $1 \mathrm{~ms}$ to 530ns. As it can be seen, the smaller the task size, the higher speedup Picos++ has when compared to the SW-only runtime. In particular Picos++ is $8 x$ and 2.5x faster than SW-only for TestFree and TestChain respectively.

\subsubsection{Support and Performance of Nested Tasks}

Figure 6 shows results of TestNested with 4 and 16 levels of nested child tasks per parent task. On the one hand, results show that Picos++ can deal with nested tasks. On the other hand, Picos++ also benefits from fine-grained nested task: with smaller task sizes Picos++ is faster than the SW-only runtime.

\subsubsection{Scalability with 15 Accelerators}

Figure 7 shows three sets of results for TestFree with 65536 tasks and with 15 dependences per task. The figures show the total execution time (bars, in the left Y-axis) of using Picos++ and SW-only runtimes and the relative speedup comparing these two (lines, in the right Y-axis). The X-axis 


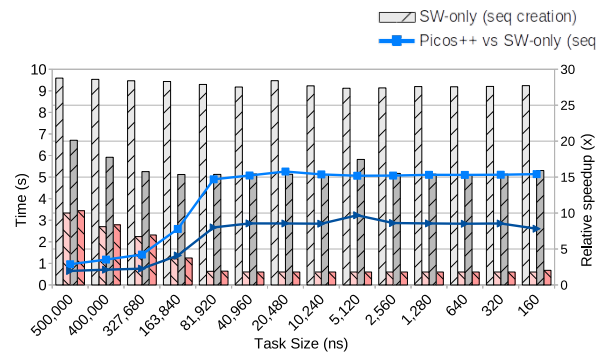

(a) Picos++ at 50Mhz

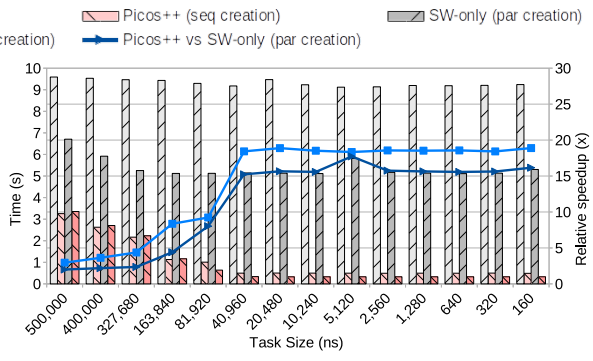

(b) Picos++ at $100 \mathrm{Mhz}$

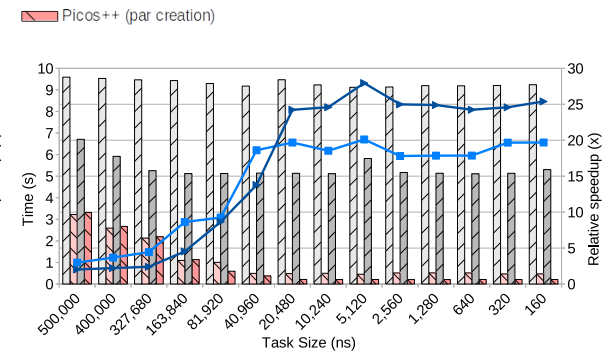

(c) Picos++ at $200 \mathrm{Mhz}$

Fig. 7: Execution time and speedup of TestFree by using Picos++ and SW-only with 15 HwAccs

in each set shows a decreasing task size from $0.5 \mathrm{~ms}$ to $160 \mathrm{~ns}$. From left to right, the three figures show Picos++ operating at 50, 100 or $200 \mathrm{MHz}$ respectively. In this experiment there are in total $15 \mathrm{HwAccs}$ operating at $200 \mathrm{MHz}$ while all the SMP cores operate at $1.1 \mathrm{GHz}$. For each set, there are also results for using either sequential or parallel task creation mechanisms in the cores.

TestFree with sequential creation used to obtain the data in Figure 7 is the same that has been used in the previous sections, where only one thread creates all the new tasks. We have realized that in this particular experiment, as there were so many (15) fast HwAccs the element limiting the execution time was in fact the task creation. In order to ease this limitation we have implemented the same program where three of the available threads create the tasks in parallel (using nested tasks). The remaining thread is used to process all the finished tasks as this is the fastest thread combination to execute this benchmark.

As it can be seen from the total execution time (bars) in Figure 7, the SW-only runtime benefits from the parallel task creation. However with task sizes smaller than $327,680 \mathrm{~ns}$ $(0.3 \mathrm{~ms})$ it is unable to execute the program faster (grey bars). On the other hand, when operating at $200 \mathrm{MHz}$, Picos++ is able to decrease the execution time with task sizes of around $10,240 \mathrm{~ns}(0.01 \mathrm{~ms})$ resulting in a $25 \mathrm{x}$ speedup against the SWonly runtime.

It can be also seen that, in the first plot where Picos++ is operating at $50 \mathrm{MHz}$, the execution time of using sequential and parallel task creation is the same. This is because there is a huge frequency gap between the cores $(1.1 \mathrm{GHz})$ and Picos++ (50MHz). Although it is around 10x faster than the SW-only runtime it is not fast enough to serve the parallel task creation with 4 cores at full speed.

Moving to the second plot where Picos++ operates at $100 \mathrm{MHz}$, it starts to take advatage of parallel creation. Now Picos++ operates at twice the frequency than the previous one and thus should obtain half of the execution time. This holds true for the parallel task creation, but not for the sequential one, which means with $100 \mathrm{MHz}$ Picos++ is able to fully serve the sequential task creation with 1 core (in this case software task creation is the bottleneck).

Finally, looking at the $200 \mathrm{MHz}$ results, the execution time with sequential task creation remains the same as expected. However, the execution time of parallel task creation is roughly one third of that with Picos++ running at $100 \mathrm{MHz}$. This means that Picos++ running at $200 \mathrm{MHz}$ is able to deal with parallel task creation with 4 cores at full speed, and the software becomes again the bottleneck. Extrapolating these results, Picos++ operating at the same speed as the cores (at $1.1 \mathrm{GHz}$ ) should be able to serve around 22 cores creating tasks at full speed with tasks as small as $1861 \mathrm{~ns}$ executing all at the same time.

To sum up briefly, Picos++ is able to take advantage of fine-grained parallelism, and it is able to achieve a $25 \mathrm{x}$ speedup over the software-only runtime with 15 HwAccs. More importantly, we can safely conclude that, with more hardware resources and a higher frequency design, Picos++ is able to achieve even higher performance for a much larger range of task granularities.

\subsection{Real Benchmarks}

\subsubsection{Performance Impact of the Task Granularity}

Figure 8 shows the speedup, power consumption, and energy savings of applications using fine-grained tasks that are executed only in SMPs. Each application in the X-axis shows two sets of problem and block size per application (their task sizes are shown in Table 1). In Y-axis the results show the results obtained by SW-only and Picos++ with 1 to 4 threads. In this experiment, Picos++ is operating at $100 \mathrm{MHz}$ and SMP is operating at $1.1 \mathrm{GHz}$.

As can be seen, Picos++ exceeds SW-only in speedup in all the cases. For example, Picos++ reaches a 3.6x speedup in Matmul with block size 64 and a 3.4x speedup in Multisort with sort size 512 . The global performance obtained when using a larger block size in Matmul and Cholesky is better because there are far less and larger tasks to manage. However this is due to the limited number of threads available in the system. With more threads Picos++ shall be able to obtain a much higher performance with block size 32 . Despite that, we can still observe that the gap between Picos++ and SW-only enlarges as the task size becomes smaller from block size 64 to 32. For example, with Matmul with block size 32 , Picos++ obtains $1.5 x$ speedup versus SWonly; Similar gap can be observed in both Cholesky and Multisort.

Figure 8 shows the power consumption of the system during Matmul execution. Other applications have similar patterns, therefore only Matmul is shown as a representative. As can be seen, the power consumption of APU is proportional to the number of threads, and that of DDR is steady during all the executions. Both SWonly and Picos++ use similar power in these two parts. For FPGA, using Picos++ consumes 0.1 watt more power. This slight consumption increase translates to the SUM (SUM=FPGA+APU+DDR) part.

Figure 8 also shows the energy consumption of all the applications by using Picos++ versus the SW-only runtime 

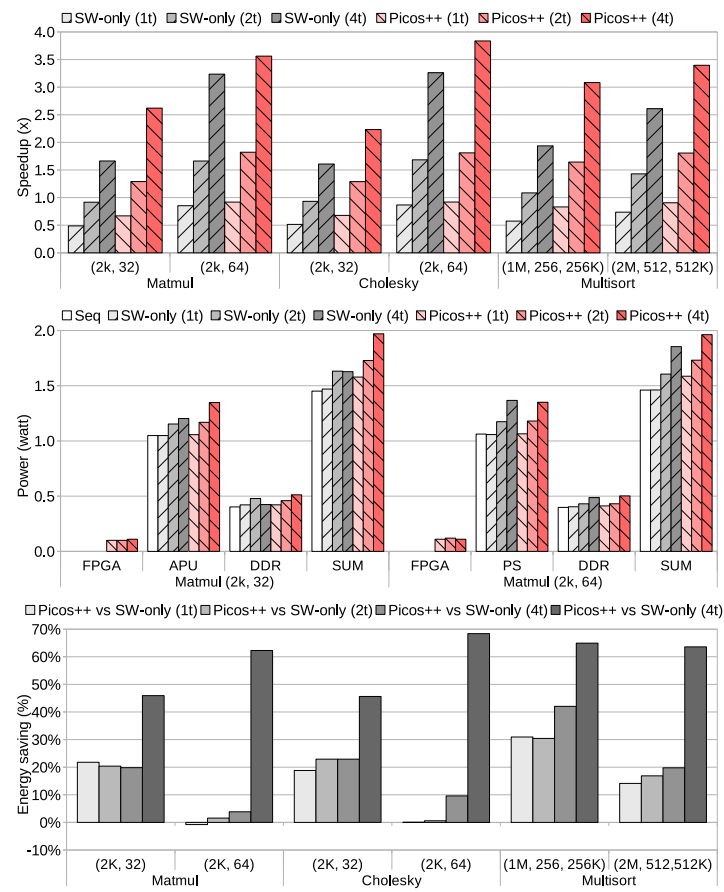

Fig. 8: Results of applications with fine-grained SMP tasks

or sequential executions. When compared to the sequential version, Picos++ runtime using 4 threads saves more than $60 \%$ of energy for all the applications, mainly due to the faster execution. When compared to the SW-only runtime, with more threads available and smaller task sizes, Picos++ saves a much higher amount of energy: $22 \%, 24 \%$ and $42 \%$ for the three applications.

Applications with medium size tasks can also benefit from using Picos++. For example, Picos++(4t) is faster executing Multisort with sort size $1 \mathrm{~K}$ with a 3.6x speedup than SW-only(4t) that only reaches a 3.1x against sequential. Correspondingly, with Multisort, Picos++(4t) saves $20 \%$ and $65 \%$ of the energy consumption compared to SW-only(4t) and sequential execution.

\subsubsection{Performance of Heterogeneous Task Scheduling}

Figures $9 \mathrm{a}, 9 \mathrm{c}$ and $9 \mathrm{e}$ show the speedup obtained in the three analyzed applications when comparing the parallel runtimes (Picos++ and SW-only) against the sequential execution. The sequential execution is obtained by using one core in the SMP. The legend SW-only(Na) indicates results obtained by using the software-only runtime with tasks that are not executed in threads but in N HwAccs. Legend SW-only $(\mathrm{Na}+4 \mathrm{t})$ indicates results obtained by using the software-only runtime with tasks that are executed in both 4 threads and N HwAccs. The same logic applies to the legends for Picos++. Special attentions should be paid for the legend with "*" symbols. For example, with Matmul block size 128 and Multisort sort size 1K in Figure 9a and 9e, it means that the system contains only 3 instead of $4 \mathrm{HwAccs}$ due to the limited capacity of the FPGA that can not hold 4 of these accelerators. It is also worth to mention that with Cholesky in Figure 9c, two different sets of HwAccs are used, hence the SW-only $(4 \mathrm{a}+4 \mathrm{t})$ and $(4 \mathrm{~g}+4 \mathrm{t})$. This will be explained later.

As can be seen, using a system with HwAccs results in a much higher speedup than using SMP threads only.
TABLE 5: The number of tasks executed in different hardware in the Picos++ system

\begin{tabular}{|c|l|lllll|}
\hline Size & Case & \multicolumn{5}{|c|}{ \#SMP \#HWACC0 \#HWACC1 \#HWACC2 \#HWACC3 } \\
\hline \multirow{3}{*}{$(2 \mathrm{k}, 32)$} & $4 \mathrm{a}$ & 0 & 41664 & 2016 & 2016 & 64 \\
& $4 \mathrm{a}+4 \mathrm{t}$ & 17992 & 23839 & 2015 & 1850 & 64 \\
& $4 \mathrm{~g}+4 \mathrm{t}$ & 4096 & 10300 & 10371 & 10451 & 10542 \\
\hline \multirow{3}{*}{$(2 \mathrm{~K}, 64)$} & $4 \mathrm{a}$ & 0 & 4963 & 496 & 496 & 32 \\
& $4 \mathrm{a}+4 \mathrm{t}$ & 2344 & 2667 & 493 & 448 & 32 \\
& $4 \mathrm{~g}+4 \mathrm{t}$ & 1024 & 1209 & 1235 & 1258 & 1258 \\
\hline
\end{tabular}

For a brief comparison, in Figure 9a Matmul Picos++(4a+4t) achieves up to 11.2x speedup while using only SMP threads (in Figure 8) the speedup obtained is only 3.6x. A similar behavior can also be observed in the other two applications.

In addition, Picos++ is much better at managing hybrid system than the software-only runtime. With tasks that can be executed in both FPGA and SMP threads, for example, for Choleksy, Picos++ $(4 a+4 t)$ reaches up to $3 x$ speedup while SW-only displays $1.7 x$. Even better, the implementation of Picos $++(4 g+4 t)$ reaches up to $6.7 x$ while the software-only runtime only achieves $4.8 x$ speedup. Similarly good results can be seen for Matmul and Multisort.

Furthermore, the gap between Picos++ and the softwareonly runtime enlarges as the task size becomes smaller (corresponding task sizes can be found in Table 2). For Matmul, the performance gap obtained when going from block size 128 to 32 enlarges from $1.1 \mathrm{x}$ to $2.7 \mathrm{x}$ faster; for Cholesky, the gap when decreasing from block size 64 to 32 goes from $1.4 \mathrm{x}$ to $2.7 \mathrm{x}$ and for Multisort, the gap grows from $1.6 x$ to $7.6 x$.

Correspondingly, Figures 9b, 9d and 9f show the energy savings of using Picos++ instead of the software-only runtime or sequential executions. As can be observed, with a smaller task size and more execution units, Picos++ saves much more energy. For example, for Matmul with block sizes 64 to 32 , Picos++ saves from up to $27 \%$ to $64 \%$ respectively; in addition with Picos++ vs SW-only(1a) to $(4 a+4 t)$, Picos++ energy saving raises from $31 \%$ to $67 \%$. With Multisort, the task sizes of 256 and 512 are very close. As a result, Picos++ saves a similar amount of energy for both granularities, up to $90 \%$. For Multisort, it can also be seen that the speedup and energy savings obtained by using 1 to 4 HwAccs do not vary much for the different task sizes. This is because that most of the execution time is spent on unraveling the nested layers. As a result there are simply not enough tasks to feed the HwAccs. With Cholesky with block size 32 , an energy saving of up to $68 \%$ of Picos++ over SW-only $(4 \mathrm{~g}+4 \mathrm{t})$ can be observed.

As mentioned earlier, two different sets of HwAccs (4a and $4 \mathrm{~g}$ ) were used for Cholesky executions shown in Figure 9c. Table 5 shows the number of tasks that have been executed in the different hardware for three cases: The first case (4a) is for using only 4 different HwAccs, corresponding to the four kernel functions of Cholesky: gemm, syrk, trsm and portf. Therefore each task can only be executed in one of these accelerators. As a result, in this case with block size 32 the software-only runtime is slower than the sequential version, however Picos++ is able to obtain a small performance improvement. The second case $(4 a+4 t)$ allows each task to be executed in one specific accelerator or in the threads. The third case $(4 \mathrm{~g}+4 \mathrm{t})$ uses four instances of the same HwAcc (gemm) and uses the threads for the 
other tasks. In the first case, there is no parallelism for the same type of tasks during execution, and the dominating number of gemm tasks also dominates the total execution time. By allowing gemm tasks to execute in SMP threads in the second case, nearly half of the gemm tasks are balanced between SMP and HwAcc0, which improves $2 x$ the performance comparing to the first case. For the third case, by employing four instances of gemm accelerators Picos++ further balances the workloads and gains a $4 x$ speedup compared to the first case.

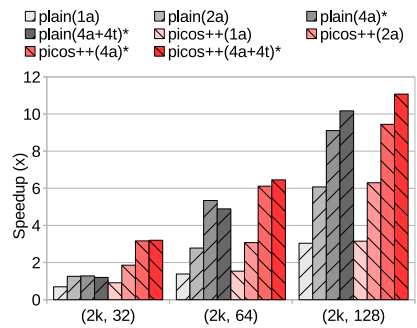

(a) Matmul speedup

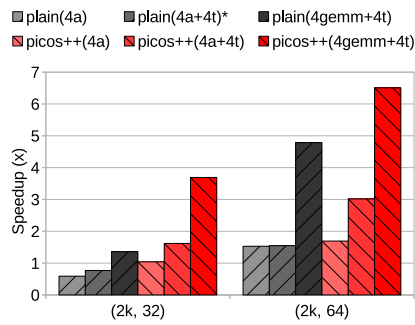

(c) Cholesky speedup

(e) Multisort speedup

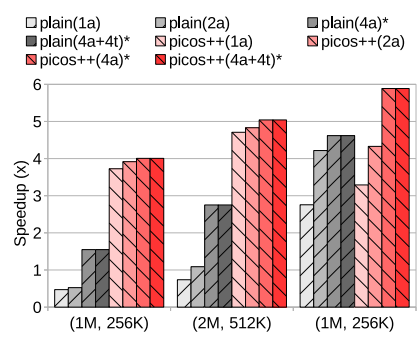

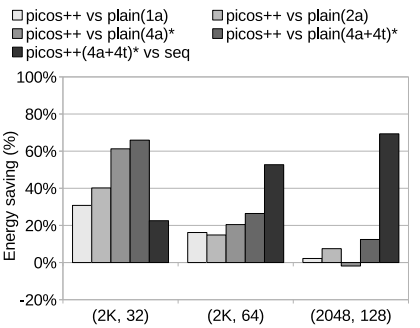

(b) Matmul energy savings

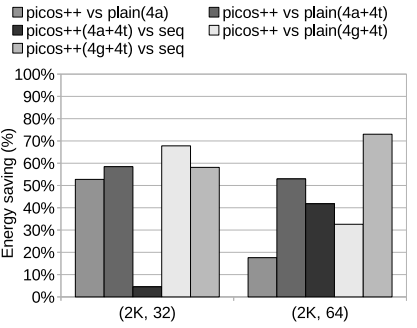

(d) Cholesky energy savings

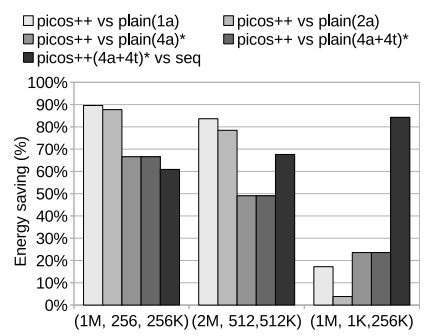

(f) Multisort energy savings
Fig. 9: Speedup and energy savings of applications

\subsubsection{Gflops and Gflops per watt}

Figure 10 shows the Gflops (Y-axis) obtained by both the software-only and Picos++ runtimes when computing Matmul with 3 HwAccs of block size 128x128 each. From left to right, and for both the software-only and Picos++ runtimes, the bars show results when using only HwAccs for task execution and HwAccs and 1, 2, 3, or 4 SMP threads to execute tasks. Tasks executed in the SMP use the optimized version of the OpenBlas sgemm (single precision Matmul) implementation. The threads operate at $1.1 \mathrm{GHz}$, Picos++ at $100 \mathrm{MHz}$ and HwAccs at $300 \mathrm{MHz}$. It is important to state that this configuraction ( 3 HwAccs of block size $128 \times 128$ ) is the one that obtains the best performance for this problem in this system when using the software-only runtime, so we selected this configuration to compare against Picos++.

The software-only runtime requires 2 threads to manage the task scheduling for 3 HwAccs, which causes perfor-

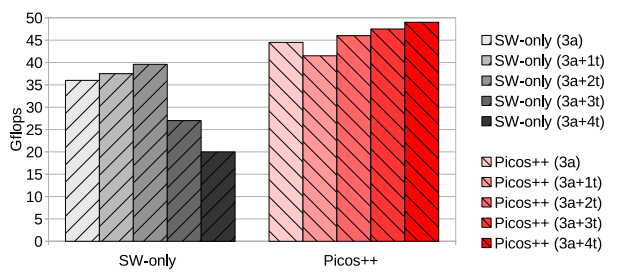

Fig. 10: Matmul Gflops with 3 FPGA accelerators

mance degration when 3 or 4 SMP threads are enabled for task execution as the same thread is used both to compute tasks and manage HwAccs. On the other hand, Picos++ increases the application performance. The small slowdown when using one additional SMP thread is due to a scheduling decision for the last task to be executed in SMP thread rather than waiting for the HwAccs. This is translated in a load unbalance between them since HwAccs are much faster than threads for task execution. However, with more threads in the system, this unbalance disappears. This unbalance with one thread could also be solved by enabling the average task execution time policy in task scheduling in Picos++ as mentioned in earlier sections.

Picos++ obtains more Gflops when more hardware resources exist in the system and better performance than the software-only runtime. The software-only runtime achieves up to 39.6 Gflops while the Picos++ runtime obtains up to 49 Gflops. The whole system (with the Picos++ runtime) executing this problem has a power consumption of only 5.74 watts, which translates into 8.54 Gflops per watt. To give an meaning to this value, the same application was run on different machines. A Intel i5-3470 core (with 4 threads at 3.20GHz) achieves 0.51 Gflops per watt. A Intel Xeon E52020 V2 (with up to 24 threads at $2.1 \mathrm{GHz}$ ) achieves 4.14 Gflops per watt and a Intel i7-4600U core (with up to 4 threads at $2.1 \mathrm{GHz}$ ) achieves 4.75 Gflops per watt.

\subsubsection{Scaling Up the Number of HwAccs}

Figure 11 shows the speedup of using Matmul with problem size $2 \mathrm{kx} 2 \mathrm{k}$ and block size $32 \times 32$ with up to 12 HwAccs (the maximum number of fmatmul HwAcc with this block size that can be fit inside the FPGA). There are two sets of results corresponding to Picos++ working at $100 \mathrm{MHz}$ and $200 \mathrm{MHz}$. For each set, there are four bars corresponding to the speedup of Picos++ over sequential and the speedup of Picos++ over the software-only runtime. In addition each case is measured with sequential and parallel task creation. The X-axis shows an increasing number of HwAccs used in the system.

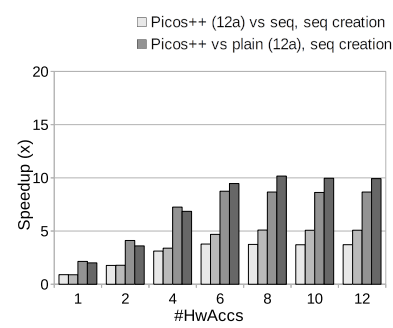

(a) Picos++ at $100 \mathrm{Mhz}$

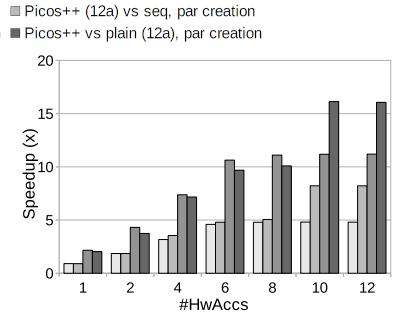

(b) Picos++ at $200 \mathrm{Mhz}$
Fig. 11: Speedup of matmul $(2 \mathrm{k}, 32)$ by using Picos ++ runtime with up to $12 \mathrm{HwAccs}$ 


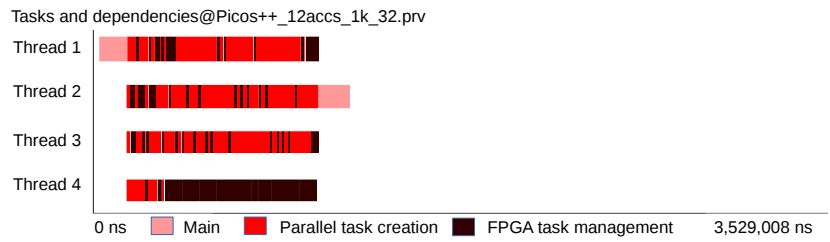

(a) by using Picos++ runtime with $12 \mathrm{HwAccs}$

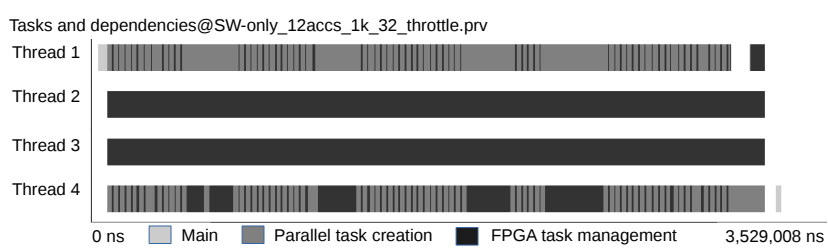

(b) by using SW-only runtime with $12 \mathrm{HwAccs}$

Fig. 12: Task Instances of Matmul 2k, 32 execution

In Figure 11, when comparing Picos++ against the sequential execution it can be seen that when executing Picos++ at $100 \mathrm{MHz}$, using parallel task creation results in a small boost of the performance of Picos++, allowing it to scale from 6 to 8 HwAccs and obtaining from $3.72 x$ to $5 x$ speedup. The applications scales up to $10 \mathrm{HwAccs}$ and the speedup improves from $4.8 \mathrm{x}$ to $8.21 \mathrm{x}$ when running with Picos++ at 200MHz. Both the size of the tasks $(27 \mathrm{~ns}$ in Table 2, corresponding to the fmatmul $32 \mathrm{HwAcc}$ at $200 \mathrm{MHz}$ ) and the maximum number of HwAccs that Picos++ is able to manage at the same time are coherent with the results shown in Figure 7. When Picos++ is compared against the software-only runtime, it can be seen that Picos++ executed at $200 \mathrm{MHz}$ and with parallel task creation achieves a $16.2 \mathrm{x}$ speedup.

To summarize, Picos++ is good at managing heterogeneous tasks when taking into account both performance and energy savings. This is partly due to the fast dependence analysis and nested task support, but also a new factor weights in. The heterogeneous task scheduling in hardware plays an important role in this case as it not only balances the workload among all the devices, but also brings the ready-to-execute tasks much closer to the HwAccs in FPGA and reduces thread contention while the software-only runtime is heavily burdened by such overhead.

To further explain this effect, Figure 12 shows two different traces of the same Matmul application (problem size and block size), with parallel task creation, using the Picos++ runtime at $200 \mathrm{MHz}$ (Figure 12a) and the softwareonly runtime at $1.1 \mathrm{GHz}$ (Figure 12b), with $12 \mathrm{HwAccs}$.

As can be seen, the execution time is much shorter when using Picos++ than the software-only runtime. When examined closely, the biggest difference between these two traces lays in the FPGA task management, as it dominates the execution time in the software-only runtime. This is because Picos++ directly manages the scheduling and finalization of FPGA tasks, therefore the FPGA task management only concerns the deletion of task descriptors in the software part of the runtime. For the software-only runtime, however, the threads are required to manage scheduling, finalization and the deletion of FPGA tasks. This overall management, which is expensive and also provokes contentions, is translated into a significant increment of the management overhead and the execution time of the application. This, together

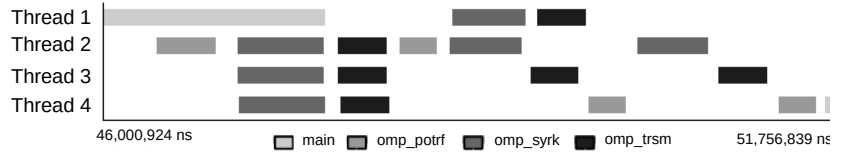

(a) Tasks instances

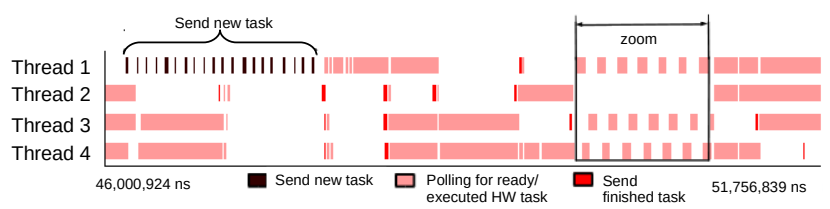

(b) Picos++ APls

Fig. 13: Visualization of Cholesky execution

with the faster task dependence analysis in hardware, explains the performance difference between the Picos++ and the software-only runtime.

\subsection{Potential Energy Savings Analysis}

Figure 13 shows an execution trace of the Cholesky application executed using Picos++ and 4 gemm HwAccs. Similar behaviors have been observed for other real applications. This trace shows, for clarity, an execution of a small problem size $256 \times 256$ with block size $64 \times 64$, which has 20 tasks in total. Figure 13a shows the tasks that each thread is executing at a given moment. As it can be seen, gemm tasks are not shown as they are executed by the HwAccs instead of by the threads. Figure 13b shows the Picos++ activities performed by each thread in the same execution. As it can be seen any thread at a given moment is executing either a SMP task, or a Picos++ activity or nothing. In addition, we have zoomed in a region in Figure 13b to show the series of sequential pollings the threads perform due to the lack of available tasks. These polling sequences are not continous.

Figure 13a shows the duration of each task: main, omp_potrf, omp_syrk and omp_trsm. Thread 1 (T.1 horizontal timeline in the figure) is the main thread and launches the main function (shown in Figure 13a), which creates new tasks (shown in the thread activity in Figure 13b), at the beginning of the trace. During this time threads 2, 3 and 4 are actively polling for ready/executed HwAcc tasks. The first SMP task that was deemed ready by Picos++ is omp_potrf, and it was scheduled and executed in thread 2 in Figure 13a. After omp_potrf, three instances of omp_syrk and then, three instances of omp_trsm are executed in threads 2, 3 and 4. Once a task finishes its execution in a thread, this thread sends a finished task to Picos++. At the end of each task in Figure 13a, it can be seen a finished task send action in Figure 13b. For all the tasks that are executed in FPGA, there is a finished HwAcc task sent from Picos++ to notify the threads to delete the task descriptor.

In Figure 13a, only a portion of time is spent executing useful work, the rest is wasted on idle polling for ready/executed HwAcc tasks as shown in Figure 13b. Table 6 quantifies the percentage of time of all the useful functions in the threads and HwAccs for a real problem with a bigger problem size $2 \mathrm{~K} \times 2 \mathrm{~K}$. As it can be seen in the Tasks category, the main task is executed mainly in Thread 1 and consumes $97 \%$ of its time. The other threads execute mainly the potrf, syrk and trsm functions when they are available. Gemm 
TABLE 6: Useful time with problem and block size $(2 \mathrm{~K}, 64)$

\begin{tabular}{|c|l|l|l|l|l|}
\hline Category & Name & T1/Acc0* & T2/Acc1* & T3/Acc2* & T4/Acc3* \\
\hline \multicolumn{1}{|c|}{ Measured percentage of useful time in an execution trace } \\
\hline \multirow{4}{*}{ Tasks } & main & $97.00 \%$ & $1.24 \%$ & $0.00 \%$ & $0.00 \%$ \\
& potrf & $0.14 \%$ & $0.78 \%$ & $0.76 \%$ & $0.75 \%$ \\
& syrk & $0.28 \%$ & $15.32 \%$ & $14.47 \%$ & $15.00 \%$ \\
& trsm & $0.26 \%$ & $20.79 \%$ & $20.79 \%$ & $22.64 \%$ \\
& gemm* & $13.34 \%$ & $56.52 \%$ & $56.52 \%$ & $60.89 \%$ \\
\hline \multirow{5}{*}{ Picos++APIs } & New task & $33.00 \%$ & 0.00 & 0.00 & 0.00 \\
& Polling ready & $0.81 \%$ & $19.19 \%$ & $19.16 \%$ & $18.49 \%$ \\
& Successful ready & $0.05 \%$ & $5.89 \%$ & $5.52 \%$ & $4.78 \%$ \\
& Finished task & $0.04 \%$ & $3.08 \%$ & $3.07 \%$ & $3.06 \%$ \\
\hline \multicolumn{6}{|c|}{ Potential energy savings time } \\
\hline HwAccs & omp_gemm* & $86.66 \%$ & $43.48 \%$ & $43.48 \%$ & $39.11 \%$ \\
Threads & Upper bound & $0 \%$ & $48.63 \%$ & $54.75 \%$ & $53.11 \%$ \\
\hline
\end{tabular}

tasks are only executed in HwAccs (HwAcc0 to HwAcc3) and do not consume any execution time in the threads but use $13.34 \%, 56.52 \%, 56.52 \%$ and $60.89 \%$ of the execution time in each HwAcc respectively.

Category Picos ++ APIs shows the time that each thread expends dealing with Picos++ activities. New task includes the time of copying necessary data from newly created Task Descriptor into the new task buffer. Finished task includes the time it takes to copy the Task Descriptor of the tasks that finished executing in threads into the finished task buffer; Polling ready includes all the time that threads spent for busy checking for ready or finished executed FPGA tasks. Successful ready shows how much time consumed for the actual successful read ready task action. As it can be seen, Polling ready is the dominant factor, due to the fact that threads have no knowledge of when there is a ready task and basically they are busy checking when there is no available parallelism.

Finally, section Potential energy savings time summarizes the percentage of the execution time in the trace that HwAccs and threads are idle, with the idea that they could be turned off to save energy without influencing performance. Picos++ has knowledge about when there is a ready task that can be executed in HwAccs or threads. Therefore, it can turn off the HwAccs and put threads to sleep. When there is work to be done, it can active HwAccs and wake up the threads. There exist well documented methods such as clock gating for turning on/off HwAccs rapidly, and commands for sleeping/waking commercial processors. Theoretically in this execution the HwAccs could be turned off for more than $40 \%$ of time. The maximum time that can be saved in each thread is computed by removing the useful functions (task creation, execution and Picos++ activities) time from the total execution time. Taking into account this upper-bound, threads 2 to 4 could be put in low power mode for approximately half of the execution time.

\subsection{Scalability analysis}

Before the hardware implementations, a software cycle-level simulator has been used to perform a design space exploration. It has measured that the baseline design with one instance of each five modules is able to scale up to 8 threads, and a bigger design with four instances of TRSs and DCTs is able to manage up to 256 threads without performance degration [13]. The current implementation of Picos++ has showed even better results in a fully operating system with 4 SMP threads and up to 15 HwAccs, which achieved up to $25 x$ speedup with TestFree and $16.2 x$ speedup Matmul application when compared with the software-only runtime.

To further support the management of more than 256 threads or different hardware devices, Picos++ should include more logic in terms of more instances of TRS and DCT modules. On one hand, using more modules linearly increases both the memory capacity of Picos++ and its internal management flow parallelism. On the other hand, although the number of modules increases the characteristics of each module remain the same, therefore the cycle time of the critical path (frequency) should not be significantly affected.

\section{Related Work}

Several papers deal with hardware support for task dependence management. Intel CARBON [19], Asynchronous Direct Messages (ADM) [20] and Task Scheduling Unit [21] introduced hierarchy hardware queue architectures to speedup task stealing and scheduling. Video-oriented task scheduler [22] and Programmable Task Management Unit (TMU) [23] extended the aforementioned works with also hardware support for task creation, synchronization and scheduling for Video processing specific applications. Multilevel Computing Architecture (MLCA) [24] introduced a novel multicore architecture for coarse-grained task parallelism for multimedia applications. The MLCA augments a traditional muticore architecture to serve as low level processing units (PU) with a high level control processor (CP). The $\mathrm{CP}$ is used for task dependence analysis and schedule tasks when they are ready. Swarm [25] uses the co-design of the execution model and micro-architecture to exploit ordered irregular task parallelism. It relies on speculative task execution and conflict detection to preserve dependences, which requires hardware transactional memory support. Fractal [26] extends Swarm to allow nested parallelism by means of task domains, that can be ordered or un-ordered to avoid over-serialization. F. Yazdanpanaha [13] proposed a task dependence manager architecture for taskbased programming models for fine-grained parallelism, studied as a cycle-level simulator. E.Castillo [7] proposed a similar hardware task-dependence manager, studied with gem5 simulator for design space exploration. Both research works proved that hardware task-dependence management can be beneficial for fine-grained parallelism. The difference is that the first work also believes in using hardware for task scheduling while the second one shows preference for software.

Nexus\# [27] and Task Superscalar [12] were also proposed to accelerate task dependence management in hardware for task-based dataflow programming models. They were both implemented in FPGA. Task Superscalar was discontinued due to some design deadlocks, and inspired Picos as an early example of a Runtime-Aware architecture [28]. On the other hand, Nexus\# was evaluated using traces of real applications and its performance results proved to be better than using software alternatives but has not been further developed. The proposal presented in this work has several new contributions to previous Picos++ work [14], in addition to be faster than its earlier alternatives [4]. In this work a new hardware support for heterogeneous task scheduling has been introduced and extended to also 
support nested tasks. The evaluation presented includes a heterogeneous system composed of 4 SMP cores plus up to 15 hardware functional units in FPGA. On the other hand, this contribution presents a method to override the limit of the original system of 15 dependences per task and the whole system has been implemented in a different hardware platform. In addition, all the energy consumption and performance results are obtained during real executions; being more significant.

Also several papers deal with heterogeneous task scheduling. Feng et al. [29] propose a system to extend OpenMP to target both SMP and GPU acelerators in software. In the area of hardware support for heterogeneous task scheduling, the Heterogeneous Earliest Finish Time (HEFT) algorithm [30] maintains a list of tasks sorted in decreasing order of their upward rank. At each schedule step, HEFT assigns the task with the highest upward rank to the processors that finishes the execution of the task at the earliest possible time. The Critical-Path-on-a-Processor (CPOP) algorithm [30] also maintains a list of tasks sorted in decreasing order as in HEFT, but in this case it is ordered according to the addition of their upward rank plus downward rank belong to the critical-path. Both of them are static algorithms. The criticality-aware task scheduler (CATS) [8] was proposed to dynamically assign critical tasks to fast cores and non-critical tasks to the slower cores in the system. The critical tasks considered are the ones in the task chain with the highest number of tasks. The criticalpath scheduler (CPATH) [8], [9] extended the consideration of task execution time for critical tasks. The version scheduler [10] described a scheduling policy to schedule tasks to either processors or GPUs in the system. Tasks that will be executed in processors or GPUs are implemented differently. The scheduling policy starts with a training stage where it learns about the different execution time in these two devices, then on the second stage it schedules tasks using the learnt information to balance the workloads. In our proposal, we borrowed this concept for heterogeneous executions implying HwAccs, in addition, the hierarchy hardware queues mentioned earlier are also a inspiration for the connection between Picos++ and different HwAccs.

\section{Conclusion}

This paper presents Picos++, a hardware runtime for task dependence management, nested task support and heterogeneous task scheduling for task-based programming models. The current Picos++ system is built on a MPSoC, which includes four symmetric ARM cores and a FPGA, both connected to the main memory. Picos++ and several hardware functional accelerators (HwAccs, for task execution) are implemented inside the FPGA with two different clock domains. Picos++ is also integrated with the taskbased programming model OmpSs, and the system runs on Ubuntu Linux 16.04.

Results of performance and energy consumption show that Picos++ is able to take advantage of high levels of finegrained task parallelism in applications, managing several execution units and heterogeneity in hardware. By comparison, the Picos++ runtime exceeds the default softwareonly runtime both in performance and energy efficiency.
With 4 threads and 4 HwAccs, Picos++ achieves up to 7.6x speedup and $90 \%$ of energy savings. With 12 HwAccs, it achieves a 16x speedup for a matrix multiplication kernel. All the factors, such as fast dependence analysis, nested task support, heterogeneous task scheduling in hardware, with the reduced thread contentions, ultimately contribute to the very high performance and energy savings gained by our proposal. For the same reasons, with more threads and hardware execution units, we can safely conclude that it will be even more significant to use Picos++ hardware runtime than a software-only one for task-based programming models.

\section{ACKNOWLEDGMENTS}

This work is supported by the Spanish Government (projects SEV-2015-0493 and TIN2015-65316-P), by the Generalitat de Catalunya (2017-SGR-1414 and 2017-SGR-1328), by the European Research Council (RoMoL GA 321253) and by the "Port of OmpSs to the Android platform and Hardware support for Nanos++ runtime" Project Cooperation Agreement with LG Electronics. We also thank the Xilinx University Program.

\section{REFERENCES}

[1] O. ARB, "Openmp application program interface - openmp standard 5," [online], 2018, https://www.openmp.org/press-release/ openmp-5-0-preview-1-published/.

[2] B. S. Center, "Ompss programming model," [online], 2016, https: //pm.bsc.es/ompss.

[3] G. Contreras and M. Martonosi, "Characterizing and improving the performance of intel threading building blocks," Workload Characterization. IEEE International Symposium, no. 57-66, 2008.

[4] X. Tan, J. Bosch et al., "Performance analysis of a hardware accelerator of dependence management for task-based dataflow programming models," International Symposium on Performance Analysis of Systems and Software (ISPASS), 2016.

[5] J. Bosch, X. Tan et al., "Characterizing and improving the performance of many-core task-based parallel programming runtimes," 31st IEEE International Parallel and Distributed Processing Symposium Workshop, 2017.

[6] N. Engelhardt, T. Dallo et al., "An integrated hardware-software approach to task graph management," in In 16th IEEE International Conference on High Performance and Communications(HPCC-2014), 2014.

[7] E. Castillo, L. Alvarez, M. Moreto et al., "Architectural support for task dependence management with flexible software scheduling," The 24th IEEE International Symposium on High-Performance Computer Architecture (HPCA'18), 2018.

[8] K. Chronaki, A. Rico, R. M. Bodia et al., "Criticality-aware dynamic task scheduling for heterogeneous system," International Conference on Supercomputing (ICS), pp. 329-338, 2015.

[9] K. Chronaki, A. Rico, M. Casas et al., "Task scheduling techniques for asymmetric multi-core systems," IEEE Transactions on Parallel and Districuted Systems (IPDPS), vol. 28, pp. 2074-2084, 2017.

[10] J. Planas, R. M. Badia, E. Ayguade, and J. Labarta, "Self-adaptive ompss tasks in heterogeneous enviroments," The 27th IEEE International Parallel and Distributed Processing Symposium (IPDPS), pp. 138-149, 2013.

[11] Y. Etsion, F. Cabarcas et al., "Task superscalar: An out-of-order task pipeline," in IEEE/ACM International Symposium on Microarchitecture (MICRO-43), 2010.

[12] F. Yazdanpanah, D. Jimenez-Gonzalez, C. Alvarez-Martinez, Y. Etsion, and R. M. Badia, "Analysis of the task superscalar architecture hardware design," Procedia Computer Science, vol. 18, pp. 339 - 348, 2013, 2013 International Conference on Computational Science. [Online]. Available: http://www. sciencedirect.com/science/article/pii/S1877050913003402

[13] F. Yazdanpanah, C. Alvarez et al., "Picos: A hardware runtime architecture support for ompss," Future Generation Computer Systems(FGCS), 2015. 
[14] X. Tan, J. Bosch et al., "General purpose task-dependence management hardware for task-based dataflow programming models," 31st IEEE International Parallel and Distributed Processing Symposium, 2017.

[15] J. Bosch, X. Tan, A. Filgueras, M. Vidal, M. Mateu, D. JimenezGonzalez, C. Alvarez, X. Martorell, E. Ayguade, and J. Labarta, "Application acceleration on fpgas with ompss@fpga," The 2018 International Conference on Field-Programmable Technology (FPT), pp. $1-8,2018$.

[16] XILINX, "Zynq ultrascale+ mpsoc overview," [online], 2017, https://www.xilinx.com/support/documentation/data_sheets/ ds891-zynq-ultrascale-plus-overview.pdf.

[17] B. S. Center, "Performance tools," [online], 2016, http://www.bsc. es/computer-sciences/performance-tools.

[18] _ "Bsc application repository," [online], 2014, https://pm.bsc. es/projects/bar/wiki/Applications.

[19] S. Kumar, C. J. Hughes et al., "Carbon: Architectural support for fine-grained parallelism on chip multiprocessors," in International Symposium on Computer Architecture, 2007.

[20] D. Sanchez, R. M. Yoo et al., "Flexible architectural support for finegrain scheduling," in International Conference on Architectural Support for Programming Languages and Operating Systems(ASPLOS), 2010.

[21] J. Hoogerbrugge and A. Terechko, "A multithreaded multicore system for embeded media processing," in Transactions on Highperformance Embeded Architectures and Compilers(THEA), 2011.

[22] G. Al-Kadi and A. S. Terechko, "A hardware task scheduler for embeded video processing," in International Conference on High Performance and Embeded Architectures and Compilers(HiPEASC), 2009.

[23] M. Sjalander, A. Terechko et al., "A look-ahead task management unit for embeded multi-core architectures," in Conference on Digital System Design(DSD), 2008.

[24] D. Capalija and T. S. Abdelrahman, "Microarchitecture of a coarsegrain out-of-order superscalar processor," in International Transaction on Parallel and Distributed Systems, 2013.

[25] M. C. Jefferey, S. Subramanian, M. Abeydeera, J. Emer, and D. Sanchez, "Data-centric execution of speculative parallel programs," Proceedings of the 49th International Symposium on Microarchitecture (MICRO), no. 1-13, 2016.

[26] S. Subramanian, M. C. Jefferey, M. Abeydeera et al., "'fractal: An execution model for fine-grain nested speculative parallelism"," International Symposium on Computer Architecture (ISCA), no. 587599, 2016.

[27] T. Dallou, A. Elhossini et al., "Nexus\#: A distributed hardware task manager for task-based programming models," in IEEE 29th International Parallel and Distributed Processing Symp(IPDPS), 2015.

[28] M. Valero, M. Moretó et al., "Runtime-aware architectures: A first approach," International Journal on Supercomputing Frontiers and Innovations, vol. 1, 2014

[29] W. Feng, B. Rountree, T. W. Scogland, and B. R. de Supinski, "Heterogeneous task scheduling for accelerated openmp," in Parallel and Distributed Processing Symposium, International. Los Alamitos, CA, USA: IEEE Computer Society, may 2012, pp. 144155. [Online]. Available: https://doi.ieeecomputersociety.org/10. 1109/IPDPS.2012.23

[30] H. Topcuoglu, S. Hariri, and M.-Y. Wu, "Performance-effective and low-complexity task scheduling for heterogeneous computing," IEEE Transactions on Parallel and Distributed Systems (IPDPS), vol. 13 , pp. $260-274,2001$.

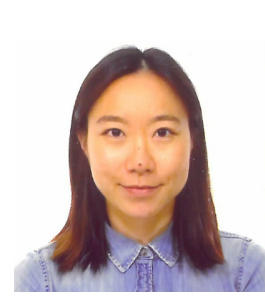

Xubin Tan received the B.S. and M.S. degrees in Electronic Information Engineering from the China Agricultural University (CAU) and in Information and Communication Engineering in Beijing Institute of Technology (BIT), Beijing in 2009 and 2012, respectively. Currently, she is is a PhD student in the department of Computer Architecture of Technical University of Catalunya (UPC) and also in the Barcelona Supercomputing Center (BSC). Her research interest lies in novel multicore, manycore, heterogeneous ar-

chitectures for high performance and low power computing

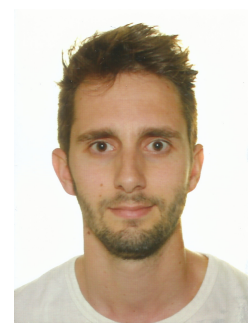

Jaume Bosch received the B.S. and M.S. degrees in Computer Science from UPC in 2015 and 2017, respectively. Currently, he is is a PhD student in the department of Computer Architecture of UPC and in the Programming Models Group in BSC. His research interest lies in parallel, distributed and heterogeneous runtime systems for High Performance Computing.

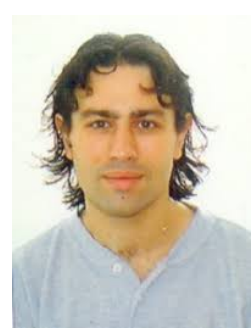

Carlos Álvarez received the M.S. and Ph.D. degrees in Computer Science from UPC, Spain in 1998 and 2007, respectively. He currently holds a position as Tenured Assistant Professor in the department of Computer Architecture at UPC, and is a associated researcher at the department of Computer Science and Programming Models at BSC. His research interests cover the areas of parallel architectures, runtime systems and reconfigurable solutions for highperformance multiprocessor systems. He has co-authored more than 40 publications in international journals and conferences. He is currently advising $1 \mathrm{PhD}$ student and has co-advised $2 \mathrm{PhD}$ theses. He has been participating in the HiPEAC Network of Excellence and in the TERAFLUX and AXIOM European projects.

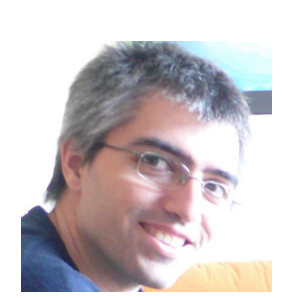

Daniel Jiménez-González received the M.S and Ph.D. degrees in Computer Science from UPC in 1997 and 2004, respectively. He currently holds a position as Tenured Assistant Professor in the department of Computer Architecture in UPC, and is a associated researcher at the department of Computer Science and Programming Models at BSC. His research interests cover the areas of parallel architectures, runtime systems and reconfigurable solutions for highperformance computing systems. He has coauthored more than 40 publications in international journals and conferences. He is currently co-advising $1 \mathrm{PhD}$ students and has co-advised $2 \mathrm{PhD}$ student. He has been participating in the HiPEAC Network of Excellence and in the SARC, ACOTES, TERAFLUX, AXIOM and PRACE European projects.

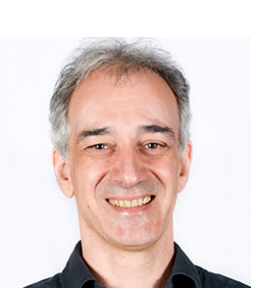

Eduard Ayguadé is a full professor in the Computer Architecture Department at UPC, lecturing on computer organization and architecture, and on parallel programming models, since 1987 His research interests cover microarchitecture, multicore architectures, and programming models and their architectural support. He has published more than 250 papers in conferences and journals in these topics. He has participated in several research projects in the framework of the European Union and research collaborations with companies. He is associated director for research on computer sciences at BSC.

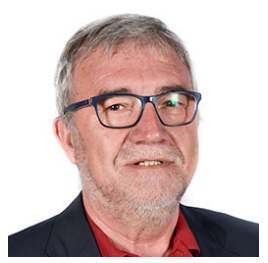

Mateo Valero is full professor at Computer Architecture Department, UPC and director at BSC. He has published 700 papers and served in organization of 300 international conferences. His main awards are: Seymour Cray, Eckert Mauchly, Harry Goode, ACM Distinguished Service, Hall of Fame member IST European Program, King Jaime I in research, two Spanish $\mathrm{Na}$ tional Awards on Informatics and Engineering. Honorary Doctorate: Universities of Chalmers, Belgrade, Las Palmas, Zaragoza, Complutense of Madrid, Granada and University of Veracruz. He is a Fellow of IEEE, ACM, and Intel Distinguished Research Fellow. He is a member of Royal Spanish Academy of Engineering, Royal Academy of Science and Arts, correspondent academic of Royal Spanish Academy of Sciences, Academia Europaea and Mexican Academy of Science. 